\title{
Incomplete Information and Incentives to Free Ride on International Environmental Resources ${ }^{1}$
}

\author{
MEHMET BAC \\ Bilkent University, Department of Economics, Bilkent, Ankara 06533, Turkey
}

R eceived J uly 27, 1994; revised O ctober 24, 1994

\begin{abstract}
This paper studies free-riding incentives on common environmental resources in an incomplete information repeated game where abatements are perishable and perfect substitutes. Two contrasting types of perfect Bayesian equilibria emerge: For high discount factors, or low discount factors but "pessimistic" prior beliefs, the inclusion of incomplete information has no impact on the pattern of abatements. Otherwise the countries attempt to use their private information strategically and the game becomes a war of attrition. This generates an outcome where the "pessimistic" country unilaterally abates forever, and another which involves delay until the first abatement if both countries are "optimistic".

(c) 1996 A cademic Press, Inc.
\end{abstract}

\section{INTRODUCTION}

International environmental resources are typically marked by the problem of transboundary externalities, absence of property rights, and individual actions of the countries that are strategic substitutes. ${ }^{2}$ Static models with these features are of the prisoners' dilemma type, therefore predict $\mathrm{H}$ ardin's [9] tragedy of commons: each country will leave it to the others to take costly actions (reduce "greenhouse gases" or pollution in the common lake) that benefit all. Dynamic complete information models have shown the opposite, notably that the repeated play of the game can alleviate the free riding problem considerably through the possibility of retaliations. ${ }^{3}$ This paper presents a dynamic game with incomplete information about valuations, an important aspect missing in the theoretical literature on transboundary pollution problems.

The importance of private information about, for instance, the relative weight of environmental problems in governments' agendas, or the private benefits and costs

${ }^{1}$ Financial support from the Beijer Institute for presentation of this paper at the research workshop in A rusha is gratefully acknowledged. I thank K arl-Göran M äler for advice and two anonymous referees for helpful criticisms and suggestions. R emaining errors and mistakes are mine.

${ }^{2}$ Loosely, individual actions are strategic substitutes if best reply functions have negative slopes. In the context of the problem of pollution control, strategic substitutability implies that a country's optimal reaction to an increase in the other country's abatement is to decrease its own. See Bulow et al. [2] for further details.

${ }^{3} \mathrm{~A}$ mong a number of recent papers using a dynamic framework to study the problem of transboundary pollution, we can cite Dockner and Long [4] who show near optimality of the steady state pollution level in a Markov perfect equilibrium if the players are patient enough and $M$ artin et al. [12] studying policy impacts of various schemes on (asymmetric) countries. Felder and $R$ utherford [5] explore the economic consequences of unilateral cutbacks of $\mathrm{CO}_{2}$ emissions. M äler [11] applies a repeated game framework to quantify the high payoffs from cooperation in the context of the E uropean acid rain game and discusses other noncooperative formulations as well. See also M äler [10] for a cooperative approach to the European acid rain game. 
of abatement stems from its potentially strategic use. Countries may choose actions or pass legislations in order to conceal their valuations. ${ }^{4}$ The inclusion of incomplete information thus enlarges the set of strategies and generates new possibilities. A high-valuation country can choose a pure defection strategy that imitates the behavior of a low-valuation country, or it can abate and reveal its valuation. A pure defection strategy can be beneficial if it induces abatements from other countries, but all are worse off if none abates. On the other hand, the consequence of revealing a high valuation is that the country cannot credibly threaten to defect in succeeding periods. A s the analysis shows, the binary decision as whether or not to reveal valuation information has more important consequences than the decision on how much to abate in a given period. $U$ sing a dynamic two-country model of transboundary pollution, we identify potential outcomes and characterize the corresponding patterns of abatements as perfect Bayesian equilibrium strategies. We provide the necessary and sufficient conditions for a high-valuation country's pure defection. The analysis also generates predictions as to the identities of the contributors and occurrence of delay until the first abatement.

This paper adopts a noncooperative approach to transboundary pollution; it is therefore closely related to the literature on private provision of public goods. The pollutants (abatements) have all the typical characteristics of public bads (goods). The "private provision" aspect, on the other hand, stems from the lack of an international "police force" to enforce agreements. Countries must provide abatements noncooperatively, as a public good is provided through the private contributions of self-interested individuals. Three types of inefficiencies are emphasized in the literature on private provision of public goods. The standard free-rider problem observed in static contexts generates the first type of inefficiency: strategic substitutability of individual contributions leads to a low level of total contributions. ${ }^{5}$ The second inefficiency takes the form of delayed contributions. It arises in dynamic settings with incomplete information and forward-looking strategic behavior. ${ }^{6} \mathrm{~A}$ third type of inefficiency arises also in dynamic settings in the form of total contributions even lower than predicted by static models. Such an equilibrium outcome involves no delay but either a few number of contributors or many contributors with very low individual contributions. Fershtman and Nitzan [6] have shown this in a dynamic model assuming complete information and continuous contributions which accumulate over time.

We show that under specific conditions the infinitely repeated game with a perishable public good (abatement of pollution) exhibits the three types of inefficiency mentioned above if valuations are private knowledge. 0 ur model generates a rich class of predictions regarding the link between initial data (prior beliefs, potential valuations of countries, and their degree of patience as represented by

\footnotetext{
${ }^{4}$ The national case studies in the M arch 1993 issue of Global Environmental Change [7] reveal various indications of this information problem, including concealing of reports by national environmental study groups.

${ }^{5}$ See, for example, Palfrey and Rosenthal [13] for a recent treatment of the classical free-rider problem in static contexts.

${ }^{6}$ The analysis of this outcome is closely related to the war of attrition games, bargaining under incomplete information (see for example Chatterjee and Samuelson [3]) and reputation (K reps and Wilson [9]). The first paper to introduce dynamics and delay costs in a noncooperative public good game is Bliss and Nalebuff [1] but their analysis is limited to a discrete public good and the game terminates at the provision date.
} 
the discount factor) and the properties of potential outcomes (efficiency, identities of the contributors, and occurrence of delay until first abatement is observed). This stems from the flexible nature of the constituent game; for some values of the parameters the constituent game turns into a chicken game, for some others, into a prisoners' dilemma. As expected, total abatements under these two types of constituent games evolve quite differently. The results and the contents of the paper are summarized below.

Section II presents a repeated game that starts under incomplete information with two potential types for the two countries. O ne type never abates, the other has an environmental valuation that exceeds abatement costs for some range of abatement levels. In general, the pattern of abatements in the overall game depends on the expected outcome of the potential subgame played under complete information. We make the standard assumption that in this subgame a "focal equilibrium" is played with symmetric and efficient payoffs subject to the subgame-perfection constraint. We then show that the perfect Bayesian equilibrium of the overall game may change dramatically as a function of these focal equilibrium payoffs which, in turn, depend on the discount factor. Equilibrium characterization, presented in Section III, reveals that for discount factors not too low the inclusion of incomplete information has no impact on the pattern of abatements, yielding a separating equilibrium in the beginning. Though this result may not be surprising (because it is in the spirit of the folk theorem), it provides insights regarding the conditions under which the countries will act cooperatively forever no matter their prior beliefs about the valuation of their opponents.

In Section IV we show that the necessary (but not sufficient) condition for the overall game to have a war of attrition outcome is a low discount factor. If the countries put much weight on their actual welfares, or, to use a public choice interpretation, if each government believes it is unlikely to govern in the next period, then the evolution of abatements depends critically on prior beliefs. Interestingly, with "impatient but pessimistic" governments the war of attrition is avoided. For other ranges of prior beliefs the inclusion of incomplete information generates an outcome exhibiting all three types of inefficiency mentioned earlier. In the resulting war of attrition game, the conceding country is the loser and abates unilaterally, while the winner thereafter chooses a pure defection strategy. This result highlights the fact that with incomplete information, more optimistic prior beliefs can actually intensify the countries' incentives to free ride and therefore lead to inefficiencies even in a repeated game setting. A $n$ interesting subclass of this outcome emerges when the discount factor is low and prior beliefs are asymmetric: With probability one, one country starts abating immediately and forever, while the other country's behavior is parasitic. Section V contains concluding remarks and interpretations of the model.

\section{THE MODEL}

We consider a two-country dynamic game of transboundary pollution where pollution (its reduction) is assumed a "Samuelsonian public bad (good)." The two countries, A and B, are each endowed with an identical private abatement technology represented by a cost function, $C(Q)$ where $Q$ denotes abatement. There are two basic types of countries. A type-L country never abates, presumably 
because its valuation is too low. The other type, called type $\mathrm{H}$, has a high valuation for the environment so that in a range of $Q$ its marginal utility exceeds marginal cost of abatement. Types are private knowledge. We make the following standard assumption:

(A 1) The utility function of type $\mathrm{H}, U(Q)$, is strictly concave and increasing and the cost function $C(Q)$ is strictly convex and increasing. Furthermore, we have $U(0)=C(0)=0$ and $\lim _{Q \rightarrow 0} U^{\prime}(Q)>\lim _{Q \rightarrow 0} C^{\prime}(Q)>0$.

This simple partial equilibrium setting can be derived from a more elaborate model in which abatement costs would be explicitly accounted for by the foregone benefit of pulling resources away from their alternative uses. The countries have the same discount factor, $0<\delta<1$, and their planning horizon is infinite. We let $\pi_{i}=\operatorname{prob}($ country $i$ is of type $\mathrm{H}$ ), for $i=\mathrm{A}, \mathrm{B}$. These probabilities are common knowledge. Given this basic structure of the model, we shall focus on the behavior of the type- $\mathrm{H}$ countries, analytically the interesting case.

In each period, the countries play a constituent game where their action sets are decomposed into two sequential moves (see Fig. 1): First, the countries decide simultaneously on whether or not to abate (participation strategy), and observe the resulting outcome. $\mathrm{N}$ ext, the countries who decided to abate decide on how much to abate (abatement strategy). Pollution is curbed by the total realized abatement and the game proceeds to the next period. ${ }^{7}$ Since a type-L country never abates, a participation with a positive abatement level, if observed, is a clear-cut signal that the country is of type $\mathrm{H}$. But if no abatement is observed, a type-H country gets a payoff of zero. Formally, the range of a mixed participation strategy $\rho_{i}$ is $[0,1]$ for $i=\mathrm{A}, \mathrm{B}$. The realization of $\rho_{i}$ is denoted $\hat{\rho}_{i}$. If a country realizes $\hat{\rho}_{i}=1$, its abatement strategy $Q_{i}$ may take any value in $R^{+}$. Clearly, $\hat{\rho}_{i}=0$ is associated with

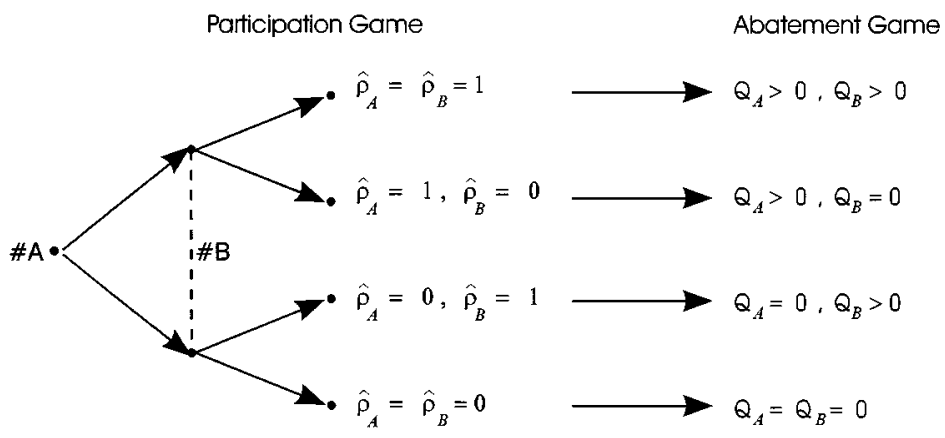

FIGURE 1

\footnotetext{
${ }^{7}$ The first justification for this decomposition is technical. It allows us to study the decision-making process sequentially: a binary participation decision and then the contribution decision. This is technically useful because dealing with randomizations over a binary participation choice when countries hesitate between participation and defection is much easier than studying randomizations over a continuum of contribution strategies. The second justification is rather intuitive. As explained below, the decision to participate (i.e., deciding to abate) is a crucial factor affecting the evolution of the game because it reveals the type of the corresponding country. Treating participation strategies separately highlights this leakage of information.
} 
$Q_{i}=0$. Since participation precedes abatement, the domain of $Q_{i}$ is restricted to the possible realization of the participation strategy.

We close this section with formal definitions of the equilibrium concept and strategies in the overall game. Let $\alpha^{t}=\left(\left\{\hat{\rho}_{A}^{t}, Q_{A}^{t}\right\} ;\left\{\hat{\rho}_{B}^{t}, Q_{B}^{t}\right\}\right)$ be the observed actions in period $t$, and define the history of the play at period $t$ by $h^{t}=$ $\left(\alpha^{1}, \alpha^{2}, \ldots, \alpha^{t-1}\right)$. In the overall game, a mixed participation strategy for country $i$ is a sequence of maps $\rho_{i}^{t}$, one for each period $t$, from possible histories $h^{t}$ into $[0,1]$. Similarly, an abatement strategy for the overall game maps $h^{t} \times\{0,1\}^{2}$ into $R^{+}$, for each $t$. These strategies may convey type-related information. At the beginning of each period, the countries use their opponent's previous participation strategies to update their beliefs according to Bayes' rule in the obvious way. Country $i^{\prime}$ 's belief in period $t$ is a function $\pi_{-i}^{t} ;\{t\} \rightarrow[0,1]$ such that, given $\pi_{i}^{t-1}$, $\rho_{i}^{t-1}$, and $\rho_{i}^{t-1}$,

$$
\pi_{-i}^{t}= \begin{cases}1 & \text { if } \hat{\rho}_{-i}^{s}=1 \text { for any } s \leq t \\ \frac{\pi_{-i}^{t-1}\left(1-\rho_{-i}^{t-1}\right)}{\pi_{-i}^{t-1}\left(1-\rho_{-i}^{t-1}\right)+\left(1-\pi_{-i}^{t-1}\right)} & \text { if } \hat{\rho}_{-i}^{s}=0 \text { for all } s<t\end{cases}
$$

Beliefs are thus required to be consistent with strategies and satisfy the "never dissuaded once convinced" condition. Observe that $\pi_{-i}$ is updated to (and will remain at) one in the period in which the other country abates. If, on the other hand, no abatement is observed, a slightly more complex updating rule is used along the equilibrium path. $N$ ote that rational countries can predict accurately the mixed strategy of their opponent along the equilibrium path. Let $V_{\mathrm{A}}^{t}$ and $V_{\mathrm{B}}^{t}$ denote respectively A's and B's expected discounted payoffs as viewed from period $t$. We require the strategies in the overall game to form a perfect Bayesian equilibrium (PBE).

A perfect Bayesian equilibrium is a pair of strategy sequences $\left\{\rho_{\mathrm{A}}^{t *}, Q_{\mathrm{A}}^{t *}\right\}$ and $\left\{\rho_{\mathrm{B}}^{t *}, Q_{\mathrm{B}}^{t *}\right\}$ of type-H countries such that, for all $t$, given the history $h^{t}$ and consistent beliefs $\left\{\pi_{\mathrm{A}}^{t}\right\}$ and $\left\{\pi_{\mathrm{B}}^{t}\right\}$,

$$
\begin{array}{ll}
V_{\mathrm{A}}^{t}\left(\left\{\rho_{\mathrm{A}}^{t *}, Q_{\mathrm{A}}^{t *}\right\},\left\{\rho_{\mathrm{B}}^{t *}, Q_{\mathrm{B}}^{t *}\right\}\right) \geq V_{\mathrm{A}}^{t}\left(\left\{\rho_{\mathrm{A}}^{t}, Q_{\mathrm{A}}^{t}\right\},\left\{\rho_{\mathrm{B}}^{t *}, Q_{\mathrm{B}}^{t *}\right\}\right) & \text { for all }\left\{\rho_{\mathrm{A}}^{t}, Q_{\mathrm{A}}^{t}\right\} \\
V_{\mathrm{B}}^{t}\left(\left\{\rho_{\mathrm{A}}^{t *}, Q_{\mathrm{A}}^{t *}\right\},\left\{\rho_{\mathrm{B}}^{t *}, Q_{\mathrm{B}}^{t *}\right\}\right) \geq V_{\mathrm{B}}^{t}\left(\left\{\rho_{\mathrm{A}}^{t *}, Q_{\mathrm{A}}^{t *}\right\},\left\{\rho_{\mathrm{B}}^{t}, Q_{\mathrm{B}}^{t}\right\}\right) & \text { for all }\left\{\rho_{\mathrm{B}}^{t}, Q_{\mathrm{B}}^{t}\right\}
\end{array}
$$

If all the elements of the sequences $\left\{\rho_{A}^{1}\right\}$ and $\left\{\rho_{B}^{1}\right\}$ are zeros and ones, we have a $\mathrm{PBE}$ in pure participation strategies. Basically, the PBE concept requires that strategies be sequentially rational (optimal given beliefs and the history of the game) and that beliefs be updated according to Bayes' rule whenever possible.

\section{THE POTENTIAL SUBGAMES AND INFORMATION STRUCTURES}

In this section, we study the equilibria of the constituent game and of some special cases of the overall game. These elements are brought together in Section IV where we present our main result. Special information structures of the overall game correspond to complete information about types, that is, $\pi_{i} \in\{0,1\}$. A mong 
the four possible combinations of these extreme prior beliefs, the case where both countries are known as type $L\left(\pi_{i}=0\right)$ is uninteresting. We therefore focus on the remaining three combinations of prior beliefs and analyze the corresponding repeated game (or any of its subgame).

The game in which it is common knowledge that country $A$ is of type $\mathrm{H}$ and country B is of type $L$ has a trivial solution: Country A abates forever with unilateral contributions given by $Q^{\mathrm{HL}}=\operatorname{argmax}_{Q}\{U(Q)-C(Q)\}$, which is unique by (A 1). We have the same outcome in the opposite case where the countries' types are reversed: Type $\mathrm{H}$ abates $Q^{\mathrm{HL}}$ forever. As we show in the sequel, the overall game has equilibria in which one country unilaterally abates forever. The subgames that have this equilibrium outcome are called the AN game or the NA game (A for abating, $\mathrm{N}$ for not abating), depending on whether it is country $\mathrm{A}$ or $\mathrm{B}$ who unilaterally abates forever. Finally, the overall game played by two type- $\mathrm{H}$ countries under complete information $\left(\pi_{i}=1\right)$, or any subgame of the overall game having this information structure, is called the AA game.

Consider now the static (one-shot) play of the constituent game where it is common knowledge that both countries are of type $\mathrm{H}$. Country $i$ 's objective is given by

$$
\max _{\left\{\rho_{i}, Q_{i}\right\}}\left\{\rho_{i}\left[\left[\rho_{j} U\left(Q_{i}+Q_{j}\right)+\left(1-\rho_{j}\right) U\left(Q_{i}\right)\right]-C\left(Q_{i}\right)\right]+\left(1-\rho_{i}\right) \rho_{j} U\left(Q_{j}\right)\right\} .
$$

This payoff function reflects the fact that, ex ante, the other country's strategies are unknown (each country has to anticipate the other country's participation and abatement). Hence, from A's viewpoint, $\rho_{\mathrm{B}}$ can be interpreted as the probability of $B$ 's participation. Notice also that individual abatements are strategic substitutes in the sense that the optimal abatement of country $i$ decreases as country $j$ increases its abatement. This is clear from the negative slope of the best reply functions $Q_{i}^{*}\left(Q_{j}\right)$ represented in Fig. 2.

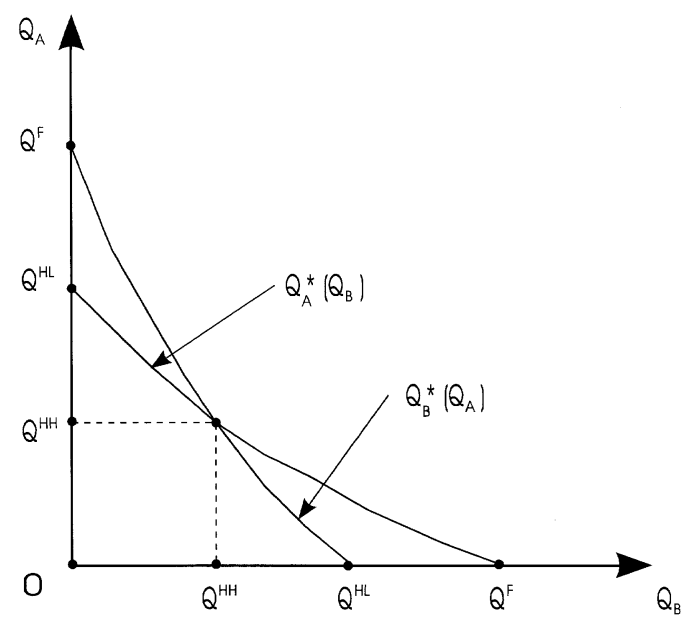

FIGURE 2 
The strongly symmetric structure of the constituent game ensures, along with the properties of the functions $U(Q)$ and $C(Q)$, a unique symmetric Nash equilibrium in which both countries participate and abate $Q^{\mathrm{HH}}=\operatorname{argmax}_{Q}\left\{U\left(Q+Q^{\mathrm{HH}}\right)-\right.$ $C(Q)$ \}. The low payoffs corresponding to symmetric abatements $Q^{\mathrm{HH}}$, denoted $\underline{P}$ in Fig. 3, represent the first type of inefficiency in the private provision of pollution reduction. As a reference point, it is useful to determine the optimum level of abatement with symmetric contributions. These solve the following problem:

$$
\max _{\left\{Q_{1}, Q_{2}\right\}}\left[2 U\left(Q_{1}+Q_{2}\right)-C\left(Q_{1}\right)-C\left(Q_{2}\right)\right]
$$

The unique symmetric solution denoted $Q^{\mathrm{F}}$ satisfies the first-order condition $2 U^{\prime}\left(2 Q^{\mathrm{F}}\right)=C^{\prime}\left(Q^{\mathrm{F}}\right)$, from which it follows that $Q^{\mathrm{F}}>Q^{\mathrm{HH}}$. We denote by $\bar{P}$ the symmetric payoff $U\left(2 Q^{\mathrm{F}}\right)-C\left(Q^{\mathrm{F}}\right)$. Thus the lower and upper bounds of total symmetric payoffs for the complete information case are respectively $2 P$ and $2 \bar{P}$.

We can now proceed with the analysis of the repeated game between two type- $\mathrm{H}$ countries under complete information. This is the AA game. From the literature on repeated games we know that the AA game has subgame perfect equilibria (SPE) in which type-H countries reach higher payoffs via the penalty of $\mathrm{Nash}$ reversion. In fact, any individually rational and feasible average payoff (up to the first-best) can be supported by SPE strategies provided that $\delta$ is sufficiently high. We shall denote by $P(\delta)=\left\{\left(P_{\mathrm{A}}(\delta), P_{\mathrm{B}}(\delta)\right) \mid P_{i}(\delta) \geq \underline{P}, i=A, B\right\}$ the set of feasible and individually rational average payoff pairs where each individual payoff is higher than the static equilibrium payoff $\underline{P}$. The set $P(\delta)$ is never empty; it gets larger as $\delta \rightarrow 1$, but $P(0)=\{\underline{P}, \underline{P}\}$ is a singleton.

A well-known problem in repeated games is the abundance of SPE. There is no agreed-upon way of choosing between the equilibria; most authors focus exclusively on an efficient equilibrium, usually a symmetric one, yet some versions of the "renegotiation proofness" concept used by a number of authors imply that the

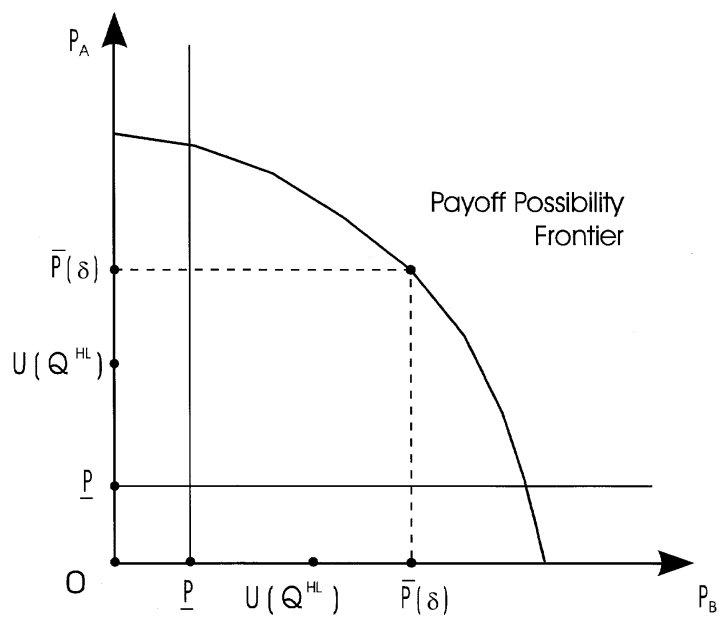

FIGURE 3 
equilibrium must be inefficient. Here we adopt the former approach widely used in the literature on repeated games. Let $\{\bar{P}(\delta), \bar{P}(\delta)\}$ denote the highest symmetric pair of payoffs that can be supported as a SPE outcome, given the discount factor. We assume that

(A 2) in the AA game, the countries play the symmetric SPE strategies associated with the highest attainable payoffs $\bar{P}(\delta)$.

M ore precisely, each country in the AA game abates $\bar{Q}(\delta)$ maximizing $[U(Q+$ $\bar{Q}(\delta))-C(Q)] /(1-\delta)$ under the subgame-perfection constraint

$$
\bar{P}(\delta) \geq(1-\delta)\left[U\left(Q^{*}(\bar{Q})+\bar{Q}(\delta)\right)-C\left(Q^{*}(\bar{Q})\right)\right]+\delta \underline{P},
$$

where $Q^{*}(\bar{Q})$ is the one-period (static) best reply to $\bar{Q}(\delta){ }^{8} \mathrm{~A}$ deviation to the best static reply yields in the actual period the payoff $U\left(Q^{*}(\bar{Q})+\bar{Q}(\delta)\right)-C\left(Q^{*}(\bar{Q})\right)$, but in all future periods the path of abatements is reversed to the static Nash equilibrium where individual payoffs are $\underline{P}$. Note that the maximum symmetric SPE payoff pair is a point on the payoff possibilities frontier in Fig. 3, and that the payoff possibilities frontier shrinks as $\delta$ decreases. If (4) holds, no country will deviate from the abatement strategy $\bar{Q}(\delta)$ supporting the payoffs $\bar{P}(\delta) \cdot{ }^{9} \mathrm{~A}$ first implication of (A 2) on the equilibria of the overall game is stated below.

PROPOSITION 1. The overall game switches immediately to the AA game no matter the priors and the discount factor if $\underline{P} \geq U\left(Q^{\mathrm{HL}}\right)$.

Proof. $P$ is the lowest attainable symmetric SPE payoff in the AA game, while $U\left(Q^{\mathrm{HL}}\right)$ is the payoff corresponding to a pure defection strategy when the other country makes its optimal unilateral abatement $Q^{\mathrm{HL}}$. Thus if $\underline{P} \geq U\left(Q^{\mathrm{HL}}\right)$, there is no incentive to conceal type information; both countries participate in the first period to reap the higher payoffs of the A A game.

Q.E.D.

Throughout the rest of the paper we shall assume that the condition in Proposition 1 does not hold. That is,

(A 3) $\underline{P}<U\left(Q^{\mathrm{HL}}\right)$.

Actually, (A 3) corresponds to the case represented in Fig. 3. The constituent game becomes a chicken game: Both countries like the mutual participation payoff $\underline{P}$ better than mutual defection, but each is strictly better off if it defects while the other country abates unilaterally. (A 3) is also a necessary but not sufficient condition for private information about valuations to be used strategically in order

\footnotetext{
${ }^{8}$ In fact, the symmetric and efficient payoffs in (A 2) can be replaced by any focal equilibrium. The argument in favor of the countries' being attracted by the focal point described in (A2) is the equal-sharing principle, quite an influential force in social contexts. Also, the payoff functions of two type-H countries are perfectly symmetric, and among the symmetric equilibria, both countries would prefer the one with highest attainable payoffs $\bar{P}(\delta)$, given the discount factor that sustains this outcome as an SPE. Note that (A 2) does not imply that the equilibrium is cooperative; all cooperation must be self-enforcing, as required by the subgame-perfection constraint (4). There is strong evidence for the power of focal points in determining outcomes, even in isolated bargaining experiments where forces favoring self-interested behavior are the most effective. See R oth [14] for a review of experiments that illustrate this phenomenon.

${ }^{9} \mathrm{M}$ äler [12] estimates the minimum discount factors required to sustain a given level of cooperation in the context of the European acid rain (this corresponds to the minimum $\delta$ that makes (4) binding). $\mathrm{He}$ also presents predictions about the countries that are most likely to deviate from a E uropean sulfur emission agreement.
} 
to extract abatements from the other country. The when and why of this strategic role of information are explored in Section IV.

Finally, consider an asymmetric information case, say, the $A N$ game where $B$ is the informed country (corresponding prior beliefs are $\pi_{\mathrm{A}}^{1}=1, \pi_{\mathrm{B}}^{1} \in[0,1)$ ). The PBE of this game is characterized below.

Proposition 2. Consider the AN game and assume (A 1), (A 2), and (A 3).

(i) If $\bar{P}(\delta) \geq U\left(Q^{\mathrm{HL}}\right)$, the $P B E$ is characterized by $\left\{\rho_{i}^{*}\right\}=\{1,1, \ldots\},\left\{Q_{i}^{*}\right\}=$ $\{\bar{Q}(\delta), \bar{Q}(\delta), \ldots\}$, with corresponding belief sequences given by $\left\{\pi_{i}\right\}=$ $\left\{\pi_{i}^{1}, 1,1, \ldots\right\}$ for $i=\mathrm{A}, \mathrm{B}$. Out-of-equilibrium-path behavior is as follows: If country $i$ deviates to $\rho_{i}^{1}=0$, country $j$ abates its best reply $Q^{\mathrm{HL}}$ until $\hat{\rho}_{i}=1$ is observed. If a deviation occurs in period $t>2$, then the static equilibrium with abatement levels $Q^{\mathrm{HH}}$ is played forever.

(ii) If $\bar{P}(\delta)<U\left(Q^{\mathrm{HL}}\right)$, the PBE is characterized by the following strategies: $\left\{\rho_{\mathrm{A}}^{*}\right\}=$ $\{1,1, \ldots\},\left\{Q_{\mathrm{A}}^{*}\right\}=\left\{Q^{\mathrm{HL}}, Q^{\mathrm{HL}}, \ldots\right\}$, and $\left\{\rho_{\mathrm{B}}^{*}\right\}=\left\{Q_{\mathrm{B}}^{*}\right\}=\{0,0, \ldots\}$. Any deviation of country $A$ is ignored, while if country $B$ deviates to $\rho_{\mathrm{B}}=1$ in any period, $\pi_{\mathrm{B}}$ is updated to one in that period and the strategies described in case (i) are played.

Proof. (i) There is no incentive to deviate from the prescribed strategies in the first period because $\bar{P}(\delta) \geq U\left(Q^{\mathrm{HL}}\right)$. H ence, both countries choose $\rho=1$ in the first period and the overall game switches to the AA game where the strategies described in the proposition are subgame perfect by definition. Hence, they are perfect Bayesian as well.

(ii) Since both types of country B play $\rho=0$ forever, A 's strategy as described in the proposition is a best reply. Country $A$ will not deviate because this merely decreases its payoff (its deviations are ignored). Nor will type $\mathrm{H}$ of $\mathrm{B}$ deviate from $\left\{\rho_{\mathrm{B}}^{*}\right\}=\{0,0, \ldots\}$ because otherwise the game collapses into the AA game where, by (A 2), the symmetric outcome yields $\bar{P}(\delta)<U\left(Q^{\mathrm{HL}}\right)$. Thus the strategies in Proposition 2 are best replies, and beliefs are consistent.

Q.E.D.

Exchanging the positions of the two countries leads to the NA game whose equilibria are exactly as described in Proposition 2. The intuition for why the informed country never abates in the equilibrium of case (ii) of Proposition 2 is quite simple: because its type cannot be verified and $\bar{P}(\delta)<U\left(Q^{\mathrm{HL}}\right)$, the informed country benefits from its informational advantage and prefers concealing its type. The threat "I will stop contributing if you (the informed country) do not contribute as well" is empty, because there is a probability (however small) that the informed country is of type L. Hence under the conditions stated in case (ii) of Proposition 2, the uninformed country is bound to abate forever. This outcome corresponds to the third type of inefficiency mentioned in the Introduction: though abatements start immediately in period one, their volume is even lower than predicted by the static, complete information version of the model.

\section{THE OVERALL INCOMPLETE INFORMATION GAME}

In this section we characterize the potential outcomes of the overall game in terms of the basic parameters: the discount factor, prior beliefs of the countries, and the payoff structure of the constituent abatement game. Before proceeding with the analysis, we mention some interpretations of the discount factor that are particularly appealing in this context. $\delta$ is usually thought of as representing pure time preference, that is, $\delta=e^{-r \Delta}$ where $\Delta$ is the length of each period of 
commitment and $r$ is the rate of time preference, or the interest rate in applications. $\delta$ increases as $r$ and /or $\Delta$ decrease. $\Delta$ can be thought of as the length of a planning period (during which the government's strategies remain fixed) while $r$ would be much affected by public choice considerations as the relative importance of other short-term objectives, employment, external balance, and so on. A nother related interpretation of $\delta$ is the probability of continuing from one period to the next, i.e., the government's prospect of remaining in office for another period. $\mathrm{O}$ ur results can easily be reformulated using the relationships between $\delta$ and the length of a period, rate of time preference, and probability of repeating the constituent game.

Since countries are diverse with respect to their rates of time preference, it is worth discussing briefly the impact of allowing for differential discount factors. This modification has a straightforward, predictable qualitative implication on the results. The symmetric SPE payoff $\bar{P}(\delta)$ corresponding to the AA game should be replaced by $\min \left\{\bar{P}\left(\delta_{\mathrm{B}}\right), \bar{P}\left(\delta_{\mathrm{A}}\right)\right\}$. This is so because it is the behavior of the "impatient" country that becomes binding in supporting the high cooperative payoffs as a SPE outcome. A n impatient country puts relatively more weight on its actual payoffs, therefore it has stronger incentives for a one-period defection from the cooperative path.

At this stage of the analysis we have to determine the necessary conditions for existence of a free-riding advantage generated purely by the incomplete information problem. We start with the following proposition which states that "pure defection forever" cannot be an equilibrium outcome if at least one country is of type $\mathrm{H}$.

PROPOSITION 3. Under (A 1), the strategy $\left\{\rho_{i}\right\}=\left\{Q_{i}\right\}=\{0,0, \ldots\}$ for $i=\mathrm{A}, \mathrm{B}$ is not a PBE strategy of a type-H country.

The proof is straightforward. Discounted payoffs of a type-H country associated with these strategies are zero, thus a deviation in any period enhances welfare. This is so because, by (A 1$)$, there exists a range of abatements such that $U^{\prime}(Q)>C^{\prime}(Q)$. Proposition 3 thus rules out the strategy pair "pure defection forever." We will show in Proposition 5 a stronger result, that an abatement will be observed with probability one at some finite date. That is, the overall game must eventually reach one of the three potential subgames among which the AN and NA game exhibit unilateral free riding through pure defection strategies. Note, however, that even if we assume (A2), which stipulates that countries play the most efficient and symmetric equilibrium in the AA game, the overall game will not necessarily collapse immediately into the AA game. The crucial parameters in this respect are explicit below in the expressions of the expected discounted payoffs of $A$ and $B$, respectively. ${ }^{10}$

$$
\begin{aligned}
V_{\mathrm{A}}^{1}= & \rho_{\mathrm{A}}^{1}\left[\rho_{\mathrm{B}}^{1} \pi_{\mathrm{B}}^{1} V_{\mathrm{A}}^{\mathrm{AA}}+\left(1-\rho_{\mathrm{B}}^{1} \pi_{\mathrm{B}}^{1}\right) V_{\mathrm{A}}^{\mathrm{AN}}\right] \\
& +\left(1-\rho_{\mathrm{A}}^{1}\right)\left[\rho_{\mathrm{B}}^{1} \pi_{\mathrm{B}}^{1} V_{\mathrm{A}}^{\mathrm{NA}}+\left(1-\rho_{\mathrm{B}}^{1} \pi_{\mathrm{B}}^{1}\right) \delta V_{\mathrm{A}}^{2}\right]
\end{aligned}
$$

\footnotetext{
${ }^{10}$ As mentioned in Section II, the strategy of the type $L$ is known, so we need only present the strategies and payoffs of type $\mathrm{H}$ when defining and analyzing equilibria. This does not imply that the countries in the game are known to be of type $H$, but rather that the behavior of type $L$ is already transparent.
} 


$$
\begin{aligned}
V_{\mathrm{B}}^{1}= & \rho_{\mathrm{B}}^{1}\left[\rho_{\mathrm{A}}^{1} \pi_{\mathrm{A}}^{1} V_{\mathrm{B}}^{\mathrm{AA}}+\left(1-\rho_{\mathrm{A}}^{1} \pi_{\mathrm{A}}^{1}\right) V_{\mathrm{B}}^{\mathrm{NA}}\right] \\
& +\left(1-\rho_{\mathrm{B}}^{1}\right)\left[\rho_{\mathrm{A}}^{1} \pi_{\mathrm{A}}^{1} V_{\mathrm{B}}^{\mathrm{AN}}+\left(1-\rho_{\mathrm{A}}^{1} \pi_{\mathrm{A}}^{1}\right) \delta V_{\mathrm{B}}^{2}\right] .
\end{aligned}
$$

The bracket following $\rho_{i}^{1}$ in the expression of $V_{i}^{1}$ represents country $i^{\prime}$ s expected discounted payoff from participating, and that following $\left(1-\rho_{i}^{1}\right)$, from defecting in period one. Combinations of $\mathrm{A}$ and $\mathrm{N}$ appear in the superscripts of $V_{i}$ to denote country $i$ 's expected discounted payoff from the three potential subgames, AA, $A N$, and NA. For example, if both countries realize participation, the game is played under complete information with average payoffs $\bar{P}(\delta)$. Thus

$$
V_{\mathrm{A}}^{\mathrm{AA}}=V_{\mathrm{B}}^{\mathrm{AA}}=\frac{\overline{\mathrm{P}}(\delta)}{1-\delta}
$$

are the discounted payoffs if both countries start abating immediately. This is a random event when mixed participation strategies are used (we omit the arguments of the strategies for conciseness). As (5) and (6) show, the probability of switching to the A A game is $\rho_{\mathrm{A}}^{1} \rho_{\mathrm{B}}^{1} \pi_{\mathrm{B}}^{1}$ from the perspective of $A$, and $\rho_{\mathrm{B}}^{1} \rho_{\mathrm{A}}^{1} \pi_{\mathrm{A}}^{1}$ from the perspective of $B$. If $A$ participates and observes no participation from $B$, then the game collapses into the AN game; $B$ free rides indefinitely. A's discounted payoffs in this case are

$$
V_{\mathrm{A}}^{\mathrm{AN}}=\frac{\left[U\left(Q^{\mathrm{HL}}\right)-C\left(Q^{\mathrm{HL}}\right)\right]}{1-\delta} .
$$

On the other hand, the opposite outcome in which $\mathrm{A}$ becomes the free rider yields

$$
V_{\mathrm{A}}^{\mathrm{NA}}=\frac{U\left(Q^{\mathrm{HL}}\right)}{1-\delta} .
$$

We have $V_{\mathrm{A}}^{\mathrm{AN}}=V_{\mathrm{A}}^{\mathrm{NA}}=V_{\mathrm{B}}^{\mathrm{AN}}$ by symmetry. ${ }^{11}$ Note that revealing a high valuation once after the other country has done so cannot be optimal because an immediate joint switching to the AA game avoids discounting, thus strictly increases payoffs. Finally, if no abatement occurs the game proceeds one period ahead. A's expected discounted payoffs are $\delta V_{\mathrm{A}}^{2}$ and B's are $\delta V_{\mathrm{B}}^{2}$.

Let us now define $\delta_{c}$, a critical value of the discount factor by

$$
\bar{P}\left(\delta_{\mathrm{c}}\right)=U\left(Q^{\mathrm{HL}}\right) .
$$

That is, if $\delta=\delta_{c}$, the highest symmetric per-period payoff that can be supported as a SPE outcome in the AA game is just equal to $U\left(Q^{\mathrm{HL}}\right)$, the static payoff from pure defection while the other country abates unilaterally. In terms of Fig. 3, recall that the payoff possibility frontier shrinks as $\delta$ falls. At precisely $\delta=\delta_{\mathrm{c}}$ the symmetric point on this frontier coincides with payoffs $U\left(Q^{\mathrm{HL}}\right)$. (A 3$)$ ensures the existence of $\delta_{c} \in(0,1)$. We can now proceed to characterize the PBE of the overall game, starting with equilibria in pure participation strategies.

\footnotetext{
${ }^{11}$ The PBE strategy profiles of the subgames AA, AN, and NA can each be thought of as a self-enforcing agreement on emissions reduction; then the overall game can be interpreted as a model of negotiation. We discuss this interpretation briefly in Section $\mathrm{V}$.
} 


\section{Proposition 4. Assume (A 1), (A 2) and (A 3).}

Case (i): $\left(\delta \geq \delta_{\mathrm{c}}\right)$. Play in the overall game switches immediately to the AA game. The corresponding PBE strategies are given in case (i) of Proposition $2:\left\{\rho_{i}^{* 1}\right\}=$ $\{1,1,1, \ldots\}, \quad\left\{Q_{i}^{1 *}\right\}=\{\bar{Q}(\delta), \bar{Q}(\delta), \ldots\}$ and beliefs form the sequence $\left\{\pi_{i}^{1}\right\}=$ $\left\{\pi_{i}^{1}, 1,1, \ldots\right\}$ for $i=\mathrm{A}, \mathrm{B}$.

Case (ii): $\left(\delta<\delta_{\mathrm{c}}\right)$. There exists a critical region of prior beliefs defined by the function $\bar{\pi}(\delta):\left[0, \delta_{\mathrm{c}}\right] \rightarrow[0,1]$, such that, if $\pi_{i}^{1} \leq \bar{\pi}(\delta)$, the equilibrium is as described in case (i) of Proposition 2. On the other hand, if $\pi_{i}^{1} \leq \bar{\pi}(\delta)<\pi_{j}^{1}$, then the following strategies constitute the unique PBE:

Country $i:\left\{\rho_{i}^{* 1}\right\}=\left\{Q_{i}^{1 *}\right\}=\{0,0, \ldots\}$

Country $j:\left\{\rho_{j}^{* 1}\right\}=\{1,1, \ldots\},\left\{Q_{j}^{1 *}\right\}=\left\{Q^{\mathrm{HL}}, Q^{\mathrm{HL}}, \ldots\right\}$.

The corresponding belief sequences are given by $\left\{\pi_{i}\right\}=\left\{\pi_{i}^{1}, \pi_{i}^{1}, \ldots\right\}$ and $\left\{\pi_{j}\right\}=$ $\left\{\pi_{j}^{1}, 1,1, \ldots\right\}$. Country $j^{\prime} s$ deviations are ignored, whereas country $i$ 's deviation to $\rho=1$ in any period leads immediately to the AA game where countries follow the SPE strategies described in Proposition 2.

Finally, if $\pi_{i}^{1}>\bar{\pi}(\delta)$ for $i=\mathrm{A}, \mathrm{B}$, no PBE in pure participation strategies exists.

Proof. Clearly, if the game has an undominated A N, NA, or a A A outcome that occurs through the play of pure participation strategies, then that outcome is bound to occur in period one. Hence, if dominated strategies are ruled out, delay may occur only through the play of mixed participation strategies. The proofs of the cases (i) and (ii) consist of deriving the best reply functions and then using these functions to construct the PBE presented in the Proposition.

(i) Consider A's best reply to $\left\{\rho_{B}^{1}\right\}=\{1,1, \ldots\}$. A has two choices in pure strategies in the first period: The first is to play $\rho_{A}^{1}=1$. If $B$ too reveals that it is of type $\mathrm{H}$, we are in the A A game where average symmetric payoffs are $\bar{P}(\delta)$. Second, country $A$ can also choose to mimic type $L\left(\rho_{A}^{1}=0\right)$ in period one, and then reveal its type or not in the second period after observing $B$ 's strategy, once it becomes the informed country. That is, $A$ can play $\left\{\rho_{A}^{2}\right\}=\{0,0, \ldots\}$ if $B$ turns out to be of type $H$, and $\left\{\rho_{A}^{2}\right\}=\{1,1, \ldots\}$ otherwise. This strategy is the best alternative to the first. Comparing these two pure strategy options leads at once to

$$
\pi_{\mathrm{B}}^{1}\left(\bar{P}(\delta)-U\left(Q^{\mathrm{HL}}\right)\right)+\left(1-\pi_{\mathrm{B}}^{1}\right)(1-\delta)\left[U\left(Q^{\mathrm{HL}}\right)-C\left(Q^{\mathrm{HL}}\right)\right] \geq 0,
$$

which states the condition under which $A$ should prefer the first option, and thus $\left\{\rho_{i}^{1}\right\}=\{1,1, \ldots\}$ for $i=\mathrm{A}, \mathrm{B}$ form a PBE. Clearly, (11) holds no matter the value of $\pi_{\mathrm{B}}^{1}$ if $\delta \geq \delta_{\mathrm{C}}$, which implies the result in case (i) by symmetry of the countries.

(ii) Consider now the case $\delta<\delta_{\mathrm{c}}$. Since $\bar{P}(\delta)-U\left(Q^{\mathrm{HL}}\right)<0$, the left hand side of (11) is decreasing in $\pi_{\mathrm{B}}^{1}$. Define the schedule $\bar{\pi}(\delta)$ by the function $\bar{\pi}(\delta):\left[0, \delta_{\mathrm{c}}\right]$ $\rightarrow[0,1]$, such that (11) holds with equality if $\pi_{\mathrm{B}}^{1}=\bar{\pi}(\delta)$. Given $\delta<\delta_{\mathrm{c}}$, we obtain the equilibrium in case (i) if $\pi_{i}^{1} \leq \bar{\pi}(\delta)$. Inspection of (11) reveals that for the case of asymmetric priors, $\pi_{i}^{1} \leq \bar{\pi}(\delta)<\pi_{j}^{1}$, the strategies described in the proposition are mutually best replies. (11) holds for country $j$ who therefore chooses $\left\{\rho_{j}^{1 *}\right\}=$ $\{1,1, \ldots\}$ no matter the strategy of country $i$. Given this strategy of $j$, the best reply of $i$ is to set $\left\{\rho_{i}^{1 *}\right\}=\{0,0, \ldots\}$ because $\bar{\pi}(\delta)<\pi_{j}^{1}$. The postulated sequences of beliefs are consistent with the strategies, and no country has any incentive to deviate given what happens off the equilibrium path. Finally, the nonexistence of a pure concession strategy equilibrium if $\bar{\pi}(\delta)<\pi_{i}^{1}$ for $i=\mathrm{A}$, B can be checked similarly making use of (11) and Proposition 3.

Q.E.D. 
To sum up and sharpen this result, with probability one, the countries reach the A A game without delay in either one of the following two cases. (i) the discount factor is high $\left(\delta \geq \delta_{\mathrm{c}}\right)$, or (ii) the discount factor is low but both prior beliefs do not exceed a critical level associated with this discount factor. The inclusion of incomplete information has no impact on the pattern of abatements in cases (i) and (ii), and countries obtain the high, cooperative, and symmetric payoffs from the beginning of the overall game. H ence, besides condition (A 3), a low discount factor is also necessary but not sufficient for the strategic use of private environmental valuations. Very asymmetric prior beliefs coupled with low discount factors may lead to the AN or NA game where the country with pessimistic prior beliefs concedes immediately and abates unilaterally in all future periods. Even if unilateral indefinite defection is individually the first-best outcome, the pessimistic country gives in and abates unilaterally. As in Proposition 2, this outcome exhibits the third form of inefficiency in the private supply of public goods: There is no delay but total per-period abatements in the A N or N A game $\left(Q^{\mathrm{HL}}\right)$ are lower than total abatements in the static play of the complete information constituent game $\left(2 Q^{\mathrm{HH}}\right)$.

A nother interesting outcome arises if both countries are impatient but none is pessimistic in the sense described above. In fact, Proposition 5 below shows that increasing the countries' optimism may intensify the problem even in a repeated game setting. However, an abatement will surely be observed at some finite date $T$ if at least one country has a high valuation for the environment.

Proposition 5. Assume (A 1)-(A 3). Let $\delta<\delta_{\mathrm{c}}$ and $\bar{\pi}(\delta) \leq \pi_{i}^{1}<1$ for $i=$ A,B. A finite integer $T>1$ and a unique $P B E$ exist such that completely mixed participation strategies $\left(\left\{\rho_{\mathrm{A}}^{t *}\right\},\left\{\rho_{\mathrm{B}}^{t *}\right\}\right)$ are played for $t<T$ as long as no participation is observed. Abatements start at date $T$ with probability one, and the PBE participation strategies for $t \geq T$ are pure and remain constant. ${ }^{12}$

Proposition 5 highlights the conditions under which the equilibrium outcome of the repeated game may involve delay until first abatement is observed: Condition (A 3) must hold, the countries must discount heavily future payoffs, and they must be optimistic as to the prospect of extracting abatements from their opponents. A long the equilibrium path, the countries revise their beliefs and put more weight on participation as the game proceeds without any abatement. Eventually, if abatements have not yet started, these beliefs fall below a critical level and a pure participation strategy is played. Of course, abatements may start earlier as a result of mixing. In each period along this equilibrium path, the expected payoff from participation equals the expected payoff from pure defection, given the other country's random participation strategy. That is, each randomization is uniquely determined by the necessity of making type $\mathrm{H}$ of the other country indifferent between participation and defection. The realized outcome may exhibit all three types of inefficiencies in the private provision of public goods.

The reason why the equilibrium path involves initially the play of completely mixed participation strategies is that each country is uncertain as to the prospect of obtaining the potential benefits of an everlasting pure defection. When countries hold optimistic beliefs about each other it is worthwhile to hold out and try extracting abatements from the opponent. Though a pure unilateral defection is

\footnotetext{
${ }^{12}$ The proof is available from the author upon request.
} 
individually the best outcome, mutual defection is the worst. A s a result, countries hesitate between pure defection and pure participation. The outcome would change dramatically if one country were informed that the other is of type $\mathrm{H}$; as in the outcome described in Proposition 2, the informed country would know the gains from defecting forever, which, if $\bar{P}(\delta)<U\left(Q^{\mathrm{HL}}\right)$, are superior to those it obtains by participating and revealing its type. Consequently, the overall game collapses into the AN or NA game where participation strategies are pure, and the country identified as type $\mathrm{H}$ abates forever. Its threat to stop abating is not credible because the informed country's type cannot be verified. Also in the overall game played under two-sided incomplete information, unilateral free-riding threats or commitments at the beginning of the game are not credible and are eliminated by the PBE concept.

\section{SUMMARY AND DISCUSSION}

Countries are diverse with respect to their environmental valuations, development plans, and short- and long-term goals. The scope and ambition of these plans or the place of environmental issues in governments' agendas can hardly be assessed from outside. Such private information has a potentially strategic use. This paper studies in a fully dynamic framework when and why informational problems are likely to cause serious inefficiencies in the control of international environmental resources. It uses an infinitely repeated game that starts under incomplete information. In each constituent game, the countries decide on whether or not to abate and, in the affirmative, on how much to abate. We solve this game for its perfect Bayesian equilibrium. Depending on prior beliefs, the discount factor, and the anticipated level of cooperation in the potential complete information subgame, we obtain the following outcomes. (i) For high discount factors, or (ii) low discount factors but pessimistic prior beliefs, the inclusion of incomplete information has no impact on the pattern of abatements. Otherwise the game becomes a war of attrition, including a potential outcome in which one country bears the burden of abating forever and another involving delay until first abatement is observed.

We can reformulate these predictions in light of the interpretations of the discount factor mentioned in Section IV. O ur results imply that if the countries interact frequently or if abatement strategies can be modified within short time periods, then the inclusion of incomplete information does not affect the pattern of abatements. With shorter periods, deviations in the complete information subgame are quickly punished, hence are more costly. As a result, higher payoffs can be supported by cooperative subgame-perfect strategies, which in turn induce highvaluation countries to reveal their types immediately. It is only when the length of a period is sufficiently high that the outcome depends critically on the distribution of prior beliefs. Immediate switching to the cooperative phase is more likely if, given the rate of time preference and length of periods, the countries are almost certain that the abatement game will never be interrupted, for instance, by international political events. On the other hand, a sufficiently high probability of interruption can lead to a war of attrition.

The overall game has an interesting alternative interpretation; it can be thought of as a model of negotiations and agreements on abatements, starting under bilateral incomplete information. A $n$ agreement can be defined as a phase of our overall game such that the PBE participation strategies are pure and remain 
constant. Since defection forever is not an equilibrium outcome, we have three potential types of agreements, which correspond precisely to the equilibria of the subgames $A A, A N$, and $N A$ : An agreement between two type- $H$ countries is reached either through mutual or unilateral concessions on abatements. N ote that the equilibria of the subgames $\mathrm{A}, \mathrm{AN}$, and NA are perfect, hence the corresponding agreements are self-enforcing with endogenized payoffs. With this interpretation, the paper offers a theory of international negotiations over bilateral emission reductions with predictions (Propositions 2, 4, and 5) as to which country is more likely to make a larger concession and whether or not delay will occur.

There are two important lines along which the paper should be extended. First, the model should be adapted to accommodate any number of countries. This seems to be a difficult task in an infinitely repeated game played under multilateral incomplete information. A nother complication with more than two countries arises if abatement strategies are not observable (this adds another source of uncertainty). Second, the level of pollution can be included as a state variable as in Dockner and Long [4] who study a differential game under complete information. If the level of pollution can increase unboundedly in the absence of abatements, type- $\mathrm{H}$ countries would be more vulnerable, and hence would have more incentives to start abating unilaterally. Besides this minor and predictable modification in the outcome, we do not expect any qualitative impact on our main results. But this conjecture has to be verified in a formal model.

\section{REFERENCES}

1. C. Bliss and B. Nalebuff, D ragon slaying and ballroom dancing: The private supply of a public good, J. Public Econom. 25, 1-12 (1984).

2. J. Bulow, J. Geanakoplos, and P. Klemperer, Multimarket oligopoly: Strategic substitutes and complements, J. Polit. Econom. 93, 488-511 (1985).

3. K. Chatterjee and L. Samuelson, Bargaining with two-sided incomplete information: An infinite horizon model with alternating offers, Rev. Econom. Stud. 54, 175-192 (1987).

4. E. J. Dockner and N. V. Long, International pollution control: Cooperative versus noncooperative strategies, J. Environ. Econom. Management 25, 13-29 (1993).

5. S. Felder and T. F. R utherford, U nilateral $\mathrm{CO}_{2}$ reductions and carbon leakage: The consequences of international trade in oil and basic materials, J. Environ. Econom. Management 25, 162-176 (1993).

6. C. Fershtman and S. Nitzan, D ynamic voluntary provision of public goods, European Econom. Rev. 35, 1057-1067 (1991).

7. Special issue on "National case studies of institutional capabilities to implement greenhouse gas reductions," Global Environmental Change, 3, no. 1 (1993).

8. G. Hardin, The tragedy of the commons, Science 162, 1243-1248 (1968).

9. D. Kreps and R. B. Wilson, R eputation and imperfect information, J. Econom. Theory 27, 253-279 (1982).

10. K. G. Mäler, The acid rain game, in "Valuation M ethods and Policy M aking in Environmental E conomics" (H. Folmer and E. van Ierland, Eds.). Elsevier Science, A msterdam (1989).

11. K. G. M äler, "The A cid R ain Game II." B eijer Discussion Paper Series N o. 32, Stockholm, Sweden (1993).

12. W. E. Martin, R. H. Patrick, and B. Tolwinski, A dynamic game of a transboundary pollutant with asymmetric players, J. Environ. Econom. Management 25, 1-12 (1993).

13. T. R. Palfrey and H. Rosenthal, Private incentives in social dilemmas, J. Public Econom. 35, 309-332 (1988).

14. A. R oth, Bargaining phenomena and bargaining theory, in "Laboratory Experimentation in E conomics: Six Points of V iew" (A. R oth, Ed.). Cambridge U niv. Press, N ew Y ork (1987). 